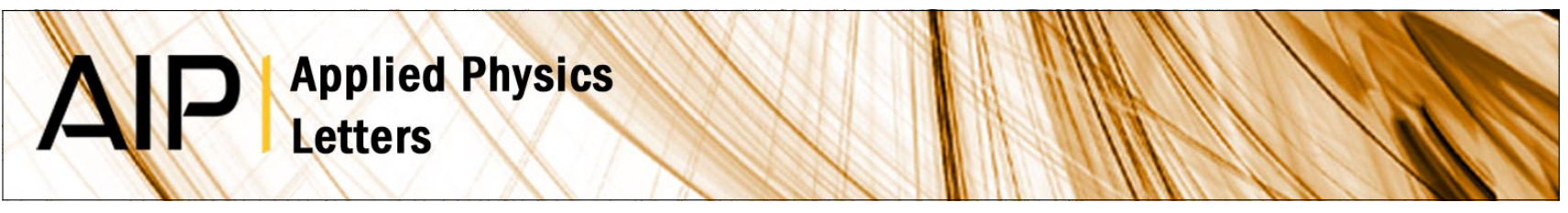

\title{
Thermal conductance of bimaterial microcantilevers
}

Sheng Shen, Arvind Narayanaswamy, Shireen Goh, and Gang Chen

Citation: Appl. Phys. Lett. 92, 063509 (2008); doi: 10.1063/1.2829999

View online: http://dx.doi.org/10.1063/1.2829999

View Table of Contents: http://apl.aip.org/resource/1/APPLAB/v92/i6

Published by the American Institute of Physics.

Additional information on Appl. Phys. Lett.

Journal Homepage: http://apl.aip.org/

Journal Information: http://apl.aip.org/about/about_the_journal

Top downloads: http://apl.aip.org/features/most_downloaded

Information for Authors: http://apl.aip.org/authors

\section{ADVERTISEMENT}

\section{AIP Applied Physics Letters}

\section{EXPLORE WHAT'S NEW IN APL}

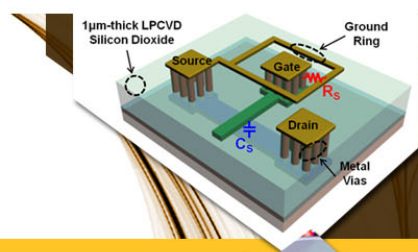

SURFACES AND INTERFACES

Focusing on physical, chemical, biological

structural, optical, magnetic and electrical

properties of surfaces and interfaces, and more...
ENERCY CONVERSION AND STORACE 


\title{
Thermal conductance of bimaterial microcantilevers
}

\author{
Sheng Shen \\ Department of Mechanical Engineering, Massachusetts Institute of Technology, Cambridge, Massachusetts \\ 02139, USA
}

Arvind Narayanaswamy ${ }^{\text {a) }}$

Department of Mechanical Engineering, Columbia University, New York, New York 10027, USA

\author{
Shireen Goh \\ Department of Materials Science and Engineering, Massachusetts Institute of Technology, Cambridge, \\ Massachusetts 02139, USA \\ Gang Chen ${ }^{\text {b) }}$ \\ Department of Mechanical Engineering, Massachusetts Institute of Technology, Cambridge, Massachusetts \\ 02139, USA
}

(Received 5 October 2007; accepted 9 December 2007; published online 13 February 2008)

\begin{abstract}
In this letter, based on the beam theory and the thermal analysis of a bimaterial cantilever, we demonstrate that the effective thermal conductance of the cantilever and the temperature at the tip of the cantilever can be determined by measuring the bending of the cantilever in response to two different thermal inputs: power absorbed at the tip and ambient temperature. (C) 2008 American Institute of Physics. [DOI: 10.1063/1.2829999]
\end{abstract}

The bimaterial cantilevers were first introduced as a calorimeter to measure the heat generated in chemical reactions. ${ }^{1}$ The same device was demonstrated to be sensitive enough to measure power as small as $100 \mathrm{pW}$ or energy of $150 \mathrm{fJ}$ in photothermal measurements. ${ }^{2}$ They were also used as IR detectors ${ }^{3-5}$ or as scanning thermal imaging probes. ${ }^{6}$ Although the bimaterial cantilevers are often used as temperature or heat flux sensors based on the beam bending due to the unequal thermal expansion of the two materials, the exact temperature at the tip of the cantilever is usually unknown. Directly measuring the temperature is difficult due to the small geometry of the cantilever structure. To find out the temperature of the cantilever, one should obtain the thermal conductance of the cantilever. However, since the thermal properties of two layers of the cantilever are dependent on their thickness, one cannot rely on theoretical calculation. In this letter, we develop a technique to determine the thermal conductance of the cantilever by measuring the bending of the cantilever in response to the variations of the absorbed power at the tip and the ambient temperature.

A triangular silicon nitride cantilever coated with $70 \mathrm{~nm}$ gold film is used in the current experiment. As shown in Fig. 1(a), a semiconductor laser beam is focused on the tip of the cantilever and reflected onto a position sensing detector (PSD). The deflection of the reflected laser beam spot on the PSD is used as a measure of the deflection of the cantilever. A part of the laser power is absorbed by the cantilever and thus creates a temperature rise at the end of the cantilever. The output of the PSD is converted into an X or Y signal corresponding to the position of the laser spot on the PSD and a sum signal proportional to the incident laser power.

The triangular shaped cantilever is simply modeled as a rectangular beam for the sake of simplicity. ${ }^{2}$ Using the beam theory, the deflection of the bimaterial strip with different

\footnotetext{
a)Electronic mail: arvind.narayanaswamy@columbia.edu.

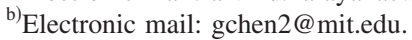

thermal expansion coefficients can be solved from the following differential equation: ${ }^{2,7,8}$

$$
\frac{d^{2} Z}{d x^{2}}=6\left(\gamma_{2}-\gamma_{1}\right) \frac{t_{1}+t_{2}}{t_{1} t_{2} K}\left[T(x)-T_{0}\right],
$$

where $Z(x)$ is the vertical deflection at a location $x, \gamma$ is the thermal expansion coefficient, $K$ is a constant defined by the thickness ratio and the Young's modulus of the layers, $t$ is the thickness of the layers $\left(t_{1}=450 \mathrm{~nm}\right.$ and $\left.t_{2}=70 \mathrm{~nm}\right), T(x)$ is the temperature distribution along the cantilever, and $T_{0}$ is the reference temperature at zero deflection. The subscripts 1 and 2 refer to the two layers: "2" for $\mathrm{Au}$ and " 1 " for $\mathrm{Si}_{3} \mathrm{~N}_{4}$. Obviously, the temperature distribution of $\left[T(x)-T_{0}\right]$ must be determined to solve the deflection $Z(x)$ from Eq. (1).

(a)

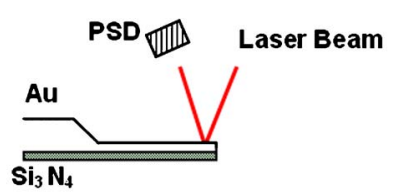

(b)
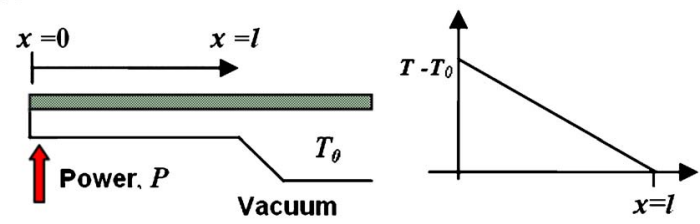

(c)

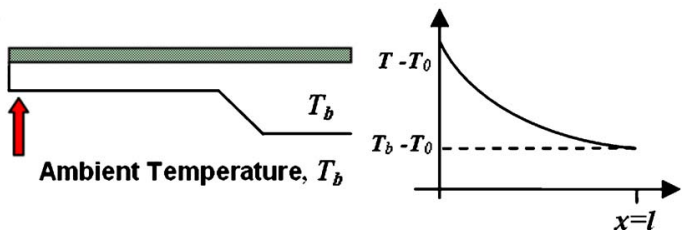

FIG. 1. (Color online) (a) The schematic drawing of cantilever, laser beam, and PSD. (b) Heating is at the end of the cantilever in vacuum. (c) The cantilever is put in a uniform temperature bath with the heating at the end. 
First, let us consider the deflection of the cantilever in vacuum when the absorbed power by the cantilever is changed. As shown in Fig. 1(b), the temperature profile in steady state is ${ }^{2}$

$$
T(x)-T_{0}=\left(1-\frac{x}{l}\right) \frac{P}{G}
$$

where $l$ is the effective length of the cantilever $(l \approx 200 \mu \mathrm{m}), G$ is the effective thermal conductance of the cantilever, and $P$ is the absorbed power. Based on the temperature distribution, the slope of the cantilever at the tip, namely, the bending angle at the tip, can be determined using Eq. (1) to be $d Z(0) / d x=-3 l P H / G$, where the constant $H$ is $H=\left(\gamma_{2}-\gamma_{1}\right)\left(t_{1}+t_{2}\right) / t_{2} t_{1} K$. As the deflection angle of the cantilever is very small, the slope at the tip is approximately equal to half the deviation angle of the reflected beam as $d Z(0) / d x=0.5 \Delta d / s$, where $\Delta d$ is the displacement of the reflected laser spot on the PSD, $s$ is the distance between the cantilever tip and the PSD. ${ }^{9}$ Thus, the deviation $\Delta d$ or the slope $d Z(0) / d x$ is what is actually measured. ${ }^{10,11}$ The sensitivity of the cantilever to the absorbed power can be theoretically expressed as $S_{P}=\partial(\Delta d) / \partial P=-6 s l H / G$. Since the thermal conductivity of thin films can be significantly lower than their corresponding bulk value, the sensitivity cannot be calculated directly. ${ }^{12}$ However, it is possible to estimate the time constant for thermal relaxation to ensure that the time scale for measurements below is long enough to reach a steady state deflection. The time constant of the cantilever was predicted to be approximately $1 \mathrm{~ms}^{2}$

In order to determine the heat absorbed by the cantilever, the absorptance of the cantilever to incident radiation needs to be known. A power meter is used to measure the radiant power in the incident beam $(1.66 \mathrm{~mW}, 650 \mathrm{~nm}$ wavelength), the reflected beam $(1.25 \mathrm{~mW})$, and the strayed beam $(0.20 \mathrm{~mW})$, resulting in an absorptance of approximately 0.126 , in agreement with published theoretical values of absorptance of similar $\mathrm{Au} / \mathrm{Si}_{3} \mathrm{~N}_{4}$ cantilevers. ${ }^{13}$ It should be noted that the scattered light by the cantilever edges is not included in the measurement and we estimate that it is less than $2 \%$ of total reflected light. During the experiments, it is not the incident but the reflected light that is measured. The ratio of the absorbed to the reflected light is 0.168 . These numbers can vary depending on the shape of the cantilever and optical arrangements.

The power output of the laser diode module can be modified by varying the operating voltage to the laser. We measured the deflection of the cantilever under the vacuum (61 $\mu$ Torr) as the power output of the laser was varied. In Fig. 2, the PSD sum signal of the reflected light is plotted as a function of the reflected laser power. The linear relationship between them corresponds to a slope of $0.6436 \mathrm{~mW} \mathrm{~V}^{-1}$. The absorption of the cantilever can be calculated from the PSD sum signal as $0.168 \times 0.6436 \times(\mathrm{PSD}$ sum signal). Finally, PSD $X$ signal versus the absorbed power is shown in Fig. 3, which gives the measured sensi-

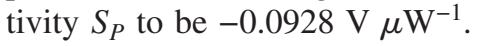

The above experiments give the deflection of the laser beam as a function of the input laser power. Next, consider the deflection of the cantilever when the incident power is kept a constant, but the ambient temperature is varied. In Fig. 1(c), the cantilever is in gaseous environment with a temperature $T_{b}$. The support of the cantilever is assumed to have

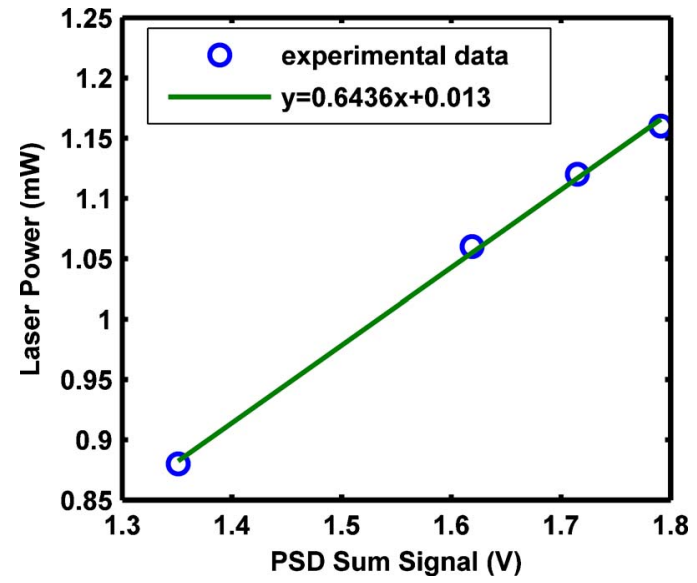

FIG. 2. (Color online) Variation of PSD sum signal with the reflected laser power.

the same temperature $T_{b}$. In this case, the cantilever still has a nonuniform temperature distribution due to incident probing laser beam at the tip, although this nonuniformity can be kept small by using a small laser power. We will show, however, that the existence of such a nonuniform temperature distribution does not matter. Since the composite layer of the cantilever is very thin compared with its length, it is treated as a "fin" with a natural convention heat transfer coefficient $h$. As shown in Fig. 1(c), the temperature profile in this case is

$$
T(x)-T_{b}=\frac{P}{G} \frac{\sinh [\beta(l-x)]}{\beta l \cosh (\beta l)},
$$

where $\beta$ is the fin parameter defined as $\sqrt{2 h\left(w+t_{1}+t_{2}\right) / l G}$ and $w$ is the effective width $(w \approx 62 \mu \mathrm{m})$. The natural convective heat transfer coefficient is predicted to be $500 \mathrm{~W} \mathrm{~m}^{-2} \mathrm{~K}^{-1}$ or even larger because of the small geometry of the cantilever. ${ }^{14}$ The corresponding slope at the end of the cantilever is given by

$$
\frac{d Z(0)}{d x}=-6 H\left\{\left(T_{b}-T_{0}\right) l+\frac{P}{G} \frac{[1-\operatorname{sech}(\beta l)]}{\beta^{2} l}\right\} .
$$

Thus, the sensitivity of the cantilever to the ambient temperature variation is obtained by $S_{T}=\partial(\Delta d) / \partial T_{b}=-12 \mathrm{sHl}$. The conductance $G$ is found to be $G=0.5 S_{T} / S_{P}$.

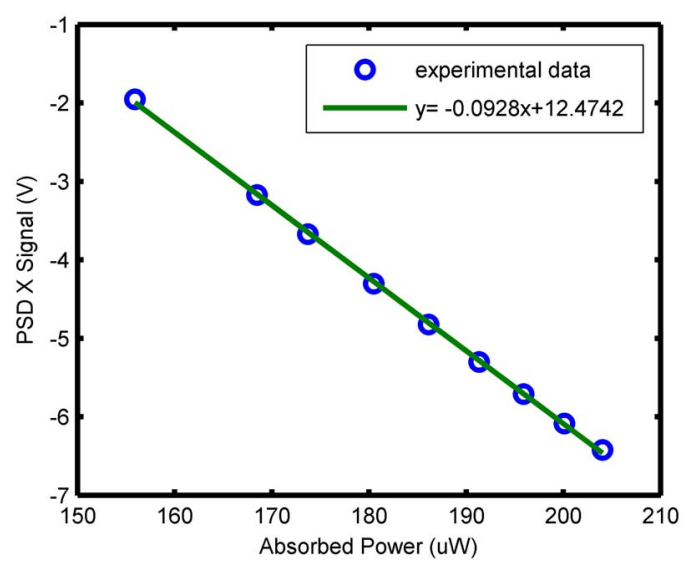

FIG. 3. (Color online) The deflection of the cantilever in vacuum due to the change of heating power. 


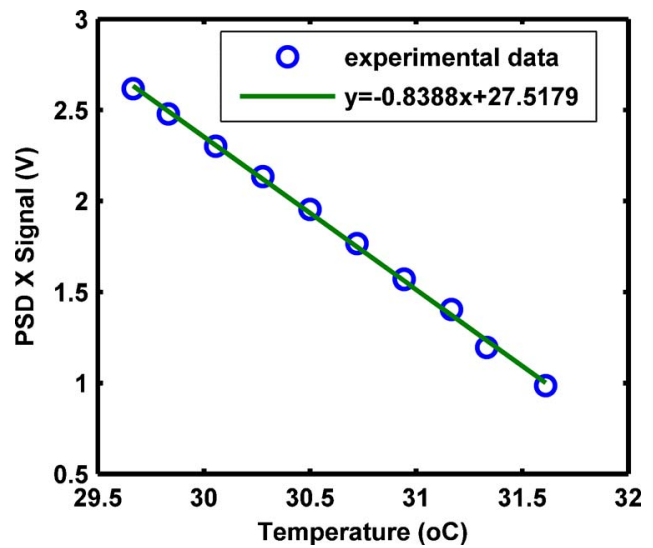

FIG. 4. (Color online) The deflection of the cantilever due to the change of ambient temperature.

To measure the sensitivity to the ambient temperature, we place the experimental setup inside a bell jar and used a hair dryer to increase the air temperature inside. Once the hair dryer is turned off, the experimental setup naturally cools. A $K$-type thermocouple is attached to the chip that holds the cantilever to measure the ambient temperature change. After an initial rapid temperature change, the temperature recorded by the thermocouple shows a slow exponential decay $\left(\leqslant 1.11 \times 10^{-3} \mathrm{~K} \mathrm{~s}^{-1}\right)$. The variation of temperature at the base is slow enough compared to the time constant of the cantilever that the steady state approximation is valid. Figure 4 shows that the PSD X signal varies with the ambient temperature. Clearly, the slope of PSD $\mathrm{X}$-temperature curve gives the sensitivity of the cantilever to the ambient temperature $S_{T},-0.8388 \mathrm{~V} \mathrm{~K}^{-1}$.

Based on $S_{P}$ and $S_{T}$, the effective thermal conductance of the cantilever $G$ is determined to be $4.50 \mu \mathrm{W} \mathrm{K}^{-1}$. Using the dimensions of the cantilever and assuming the thermal conductivities of the $\mathrm{Si}_{3} \mathrm{~N}_{4}$ (Ref. 15) and $\mathrm{Au}$ (Ref. 16) films to be 2.5 and $190 \mathrm{~W} \mathrm{~m}^{-1} \mathrm{~K}^{-1}$, respectively, the theoretical value of conductance is estimated to be around $5.88 \mu \mathrm{W} \mathrm{K}^{-1}$. We point out that the thermal conductivities of $\mathrm{Si}_{3} \mathrm{~N}_{4}$ and $\mathrm{Au}$ films used here are smaller than their bulk values due to grain-boundary and boundary scatterings. The agreement between measurement and estimation is reasonable as the exact values of the thermal conductivities for both layers are not known accurately. Once the power absorbed by the cantile- ver is known, the temperature difference between the tip and the ambient can be obtained from Eq. (2). For example, when the PSD sum signal of the reflected light is $1.80 \mathrm{~V}$, the absorbed power is given by $0.168 \times 0.6436 \times 1.80$ $\approx 194.6 \mu \mathrm{W}$, and the temperature difference is $43.1 \mathrm{~K}$.

In summary, we use simple beam theory and the thermal analysis of a bimaterial cantilever to demonstrate that the effective thermal conductance of the cantilever can be determined by measuring the sensitivities of the cantilever to variations in the absorbed power and the ambient temperature. This method does not rely on the knowledge of the geometric parameters of the cantilever, such as the length or thickness of either layer, which could vary from cantilever to cantilever. Our experimental results on a $\mathrm{Si}_{3} \mathrm{~N}_{4} / \mathrm{Au}$ bilayer cantilever are in agreement with expectations.

The authors wish to thank Austin Minnich and Lu Hu for their advice. This work was supported by DOE (Contract No. DE-FG02-02ER45977) and NSF (CTS-0506830).

${ }^{1}$ J. K. Gimzewski, Ch. Gerber, E. Meyer, and R. R. Schlittler, Chem. Phys. Lett. 217, 589 (1994).

${ }^{2}$ J. R. Barnes, R. J. Stephenson, C. N. Woodburn, S. J. O’Shea, M. E. Welland, T. Rayment, J. K. Gimzewski, and Ch. Gerber, Rev. Sci. Instrum. 65, 3793 (1994).

${ }^{3}$ J. R. Barnes, R. J. Stephenson, M. E. Welland, Ch. Gerber, and J. K. Gimzewski, Nature (London) 372, 79 (1994).

${ }^{4}$ J. Varesi, J. Lai, T. Perazzo, Z. Shi, and A. Majumda, Appl. Phys. Lett. 71, 306 (1997).

${ }^{5}$ P. G. Datskos, P. I. Oden, T. Thundat, E. A. Watchter, R. J. Warmack, and S. R. Hunter, Appl. Phys. Lett. 69, 2986 (1996).

${ }^{6}$ O. Nakabeppu, M. Chandrachood, Y. Wu, J. Lai, and A. Majumda, Appl. Phys. Lett. 66, 694 (1995).

${ }^{7}$ R. J. Roark and W. C. Young, Formulas for Stress and Strain, 5th ed. (McGraw-Hill, New York, 1975).

${ }^{8}$ J. Lai, T. Perazzo, Z. Shi, and A. Majumda, Sens. Actuators, A 58, 113 (1997).

${ }^{9}$ A. M. Moulin, R. J. Stephenson, and M. E. Welland, J. Vac. Sci. Technol. B 15, 590 (1997).

${ }^{10}$ H. J. Butt and M. Jaschke, Nanotechnology 6, 1 (1995).

${ }^{11}$ R. Lévy and M. Maaloum, Nanotechnology 13, 33 (2002).

${ }^{12} \mathrm{G}$. Chen, Nanoscale Energy Transport and Conversion (Oxford, New York, 2005).

${ }^{13}$ A. Wig, A. Passian, E. Arakawa, T. L. Ferrell, and T. Thundat, J. Appl. Phys. 95, 1162 (2004).

${ }^{14}$ A. F. Mills, Heat Transfer, 2nd ed. (Prentice-Hall, Engleweed Cliffs, NJ, 1999).

${ }^{15}$ D. Song, Ph.D. thesis, University of California, Los Angeles, 2003.

${ }^{16}$ G. Langer, J. Hartmann, and M. Reichling, Rev. Sci. Instrum. 68, 1510 (1996). 